\title{
Multiphase Flows in Materials Processing: The Road Map for Modeling, Experimentation, Validation, and Optimization
}

\author{
KINNOR CHATTOPADHYAY ${ }^{1,2}$ \\ 1.-Process Metallurgy Research Labs, Department of Materials Science and Engineering, Cross \\ Appointed toMechanical and Industrial Engineering, University of Toronto, 184 College Street, \\ Suite 140, Toronto, ON M5S3E4, Canada. 2.-e-mail: kinnor.chattopadhyay@utoronto.ca
}

Most operations in materials processing involve complex phenomena comprised of chemical thermodynamics and associated momentum, heat, and/or mass transport, and these apply to ferrous processes, non-ferrous processes, casting of metals, atomization of metals to produce powders and nano-powders, welding and joining processes, special refining techniques, etc. All these processes essentially comprise of turbulent non-isothermal reacting multiphase flows. As difficult as it sounds, researchers often start with chemical thermodynamics to understand the feasibility of a process. While thermodynamics is an excellent tool to start with, it only tells us half the story, and thus transport phenomena, i.e., fluid flows, heat transfer and mass transfer, play a dominant role in materials processing since their respective rules dictate the kinetics of the various physical phenomena occurring in these processes. These phenomena include such events as multi-phase reactions, entrainment of secondary and tertiary phases in the primary phase (slag and gas entrainment in liquid metals), degassing of metals and alloys, alloy melting and mixing, the movements and flotation of particles in melts, melt temperature losses, residence times in metallurgical reactors, erosion of refractory linings, particle size distribution during high pressure atomization, etc. These are complicated multiphase processes and often include multiphysics-based phenomena. Hence, it is of utmost importance that the development and improvement in our multiphase flow research techniques, and our abilities to simulate these flows and their associated transport phenomena, contribute significantly to our knowledge-building endeavors, which in turn help in effectively designing and operating

Kinnor Chattopadhyay is the JOM advisor for the Process Technology and Modeling Committee - part of the TMS Extraction \& Processing Division and the Materials Processing \& Manufacturing Division - and guest editor for the topic Multiphase Flows in Materials Processing: Part I in this issue. metallurgical/materials processes. Over the last three decades, researchers have used different tools, including computational fluid dynamics (CFD) simulation, followed by experimental validation, and finally comparing with real process data to optimize these complex materials processes.

This special $J O M$ topic is focused on multiphase flows in materials processing, and contributors from around the world have been encouraged to submit articles related to multiphase flow modeling techniques, experimental techniques in multiphase flows, and CFD modeling of metallurgical processes involving multiphase flows. In the current issue, researchers have contributed on modeling of multiphase flows for a variety of metallurgical processes, including continuous casting, ladle metallurgy, copper converting, basic oxygen steelmaking, electroslag re-melting, and selective laser melting. Some of the key phenomena considered in this particular issue include bubble expansion, two-phase mixing, effects of various interfacial forces, electromagnetic effects and finally solidification. These phenomena are complicated in nature, and the contributors have done an excellent job in explaining the details of their modeling techniques so that their methods can be cross-pollinated to other areas of materials processing, from which the readers will benefit. Another important aspect to note is that most of the contributing authors have taken an industrial metallurgical process under consideration, and this also adds value to our industrial readers.

Process simulations using CFD codes can give detailed information of fluid flow, heat and mass transfer in materials process and metallurgical reactors, and this can lead to a better understanding of a given process. During the past three decades, the range and power of CFD codes have been considerably developed, and CFD simulations have been extensively applied to help in understanding a given process and in guiding new process developments. Thanks to the genius and efforts of 
Professor Spalding and co-workers at Imperial College, London, a generalized transport equation had been formulated, which has been used to describe all manner of transport phenomena. This has been a great help in obtaining iterative solutions to a series of partial differential equations requiring simultaneous solutions. In turn, it has spawned the formulation of general purpose computer codes that have become increasingly user friendly and robust. Then, in the 1970s, Launder and Spalding introduced the famous $k$-e model for turbulent flows which represented yet another breakthrough for solving turbulent flow problems. Later, other researchers introduced variants of this model, such as the renormalization group $k-\mathrm{e}$ model and the realizable $k-\mathrm{e}$ model. For tackling the boundary problems in turbulent flows, some researchers developed the low Re number $k$-e model using damping factors. Launder and Spalding also gave the concept of wall functions for boundary treatments. Patankar and Spalding paved the way for solving pressure-linked equations for the first time by introducing the famous SIMPLE algorithm. Later on, other algorithms such as SIMPLER, SIMPLEST, SIMPLEC, PISO, etc., were proposed by various researchers. Based on the above concepts, many software packages, such as PHOENICS, CFX, FLOW-3D, FIDAP, FLUENT, COMSOL, etc., were marketed, and have allowed CFD to become an increasingly common tool used by non-experts. However, it is essential that one learns the physics behind these software packages before blindly using them. Multiphase flow modeling techniques have also evolved significantly over the last couple of decades. Initially, researchers would simplify the problems by considering the dominant type of flow, and would be interested either in particle tracking or in interface tracking or just in interactions between the phases in a mixture. However, recently, researchers have started to use hybrid techniques to account for different types of flows in a single system, including dispersed, segregated, particulate and continuous phases. Also, the growing popularity of open source codes have encouraged researchers to test and try out different hybrid techniques and numerical schemes. Finally, a number of researchers have started coupling chemical reactions with multiphase flows to make the simulations more realistic.

While simulations are an excellent way to understand multiphase flows in materials processes, there needs to be a validation step to confirm whether the simulations are robust and can faithfully represent the real process. As the quality and accuracy of instruments have significantly improved in the last two decades, researchers have exploited this advantage by incorporating experimentation in their studies. Tools like a time-resolved, multiphase PIV (particle image velocimetry) system, ultrasound Doppler velocimetry (UDV) system, and high-speed cameras have contributed greatly in validating multiphase CFD models. Researchers have even gone further by using flexible grid PIV techniques, and using these, much like the way CFD works, the philosophy is to create calculation grids, which have been adapted to the flow and the geometry of what is measured. A grid object is a user-specified region, in which calculation points are distributed in a way the user desires. Furthermore, the user can specify different calculation methods to be applied to different objects and regions. Flexible grid PIV has become an ideal tool for comparing experimental flow data with computational data, as they use the same grid size and resolution for both. Also, modern PIVs have the capabilities to measure flows in multiphase systems and generate valuable information on flow behavior. Some unique features of the multiphase PIVs systems are that the technique is non-invasive and measures the velocities of micro-particles and secondary phases following the flow. It employs fluorescent particles as flow tracers for the liquid phase and optical filters to see the secondary phase separately in the experiment. If simultaneous data from both phases are required, it is generally necessary to have two cameras where the images of the region of interest are combined with special optics. The UDV technique has also proved to be a very efficient tool for measuring velocities in opaque liquids, especially for measuring bubble velocities and slurry type flows. Some researchers also perform multiphase experiments in high-temperature environments with opaque metals, and the UDV technique has enabled measurements for such systems, and also let us compare the data with multiphase CFD simulations.

As the industries involved in materials processing have continuously invested in $R \& D$, they have also become open to share real process data with researchers, and that has provided a realistic validation milestone for physical and mathematical models involving multiphase flows. While the importance of the synergy between modeling, experiments and real plant data has been emphasized by many, today we can be proud of the fact of its actual implementation. Recently, researchers have also emphasized on the importance of the "knowledge and insight of the modeler", and the need to involve "multidisciplinary teams with process and computational backgrounds" to hunt for the truth behind a process and to optimize it. The ability to capture a significant amount of process data, and subsequently use data analytics and machine learning techniques, will be adding a new dimension to the validation and optimization process. While simulations using CFD models and physical models generate data for statistical and sensitivity analyses, they can now be compared with plant data in real time. Also, integrating machine learning platforms with simulations is going to give researchers an opportunity to feed boundary conditions into multiphase flow models in real time. 
The advent of CFD, and advanced experimental instruments, coupled with real process data and machine learning will create a revolution in the field of multiphase flows in materials processing, and in materials process optimization. These types of approaches will allow researchers to challenge their limits and go into a world beyond imagination. Process models validated with actual experiments have proved to be reliable for the last three decades, and this will surely continue by the incorporation of additional features such as machine learning and data analytics. The motivation for this will be the continuing quest for knowledge which will be aided by ever-increasing computational power and exponential decrease in computational costs for realtime analytics, validation and optimization.

The following list summarizes the papers being published under the topic of "Multiphase Flows in Materials Processing: Part I". To download any of the papers, follow the URL http://link.springer.com/ journal/11837/70/10/page/ 1 to the table of contents page for the October 2018 issue (vol. 70, no. 10).

- "Modeling of Mixing Behavior in a Combined Blowing Steelmaking Converter with a FilterBased Euler-Lagrange Model" by Mingming Li, Lin Li, Qiang Li, and Zongshu Zou.

- "Fluid Flow and Solidified Shell Remelting in the F-EMS Area During Billet Continuous Casting" by Wenxiang Jiang, Mujun Long, Tao Liu, Dengfu Chen, Huabiao Chen, Junsheng Cao, Helin Fan, Sheng Yu, and Huanmei Duan.

- "A Water Model Study on Mixing Behavior of the Two-Layered Bath in Bottom-Blown Copper Smelting Furnace" by Lang Shui, Zhixiang Cui, Xiaodong Ma, Xu Jiang, Mao Chen, Yong Xiang, and Baojun Zhao.

- "Mathematical Modeling of the Multiphase Flow and Mixing Phenomena in a Gas-Stirred Ladle: The Effect of Bubble Expansion" by Qing Cao and Laurentiu Nastac.
- "A Study on the Influence of Scanning Strategies on the Levelness of the Melt Track in Selective Laser Melting Process of Stainless Steel Powder" by Kim Quy Le, Chao Tang, and Chee How Wong.

- "Effects of Angle of Rotation of Submerged Entry Nozzle on Fluid Flows in a Square Billet Casting Mold" by Mahdi M. Aboutalebi, Francis Lapointe, Julien D'amours, Mihaiela M. Isac, and Roderick I.L.Guthrie.

- "Effect of Current Density on Metal Droplet Dropping and Solidification in ESR System" by Xiaohua Wang and Ying Li.

- "Control of Two-Phase Flows During Startup Operations of Casting Sequences in a Billet Tundish" by R.D. Morales, Javier Guarneros, A. Nájera-Bastida, and Jafeth Rodriguez.

- "Numerical Modeling of Liquid-Liquid Mass Transfer and the Influence of Mixing in GasStirred Ladles" by Quynh N. Hoang, Marco A. Ramírez-Argáez, Alberto Conejo, Bart Blanpain, and Abhishek Dutta.

- "An Investigation of the Behavior of the Surficial Longitudinal Wave in a Bottom-Blown Copper Smelting Furnace" by Lang Shui, Xiaodong Ma, Zhixiang Cui, and Baojun Zhao.

- "Effects of Interphase Forces on Fluid Flow in Gas-Stirred Steel Ladles Using the Euler-Lagrange Multiphase Approach" by Haojian Duan, Ying Ren, and Lifeng Zhang.

- "Transient, Multiphase Simulation of Grade Intermixing in a Tundish Under Constant Casting Rate and Validation Against Physical Modeling" by Krshnavtar and Dipak Mazumdar.

- "Modeling Argon Gas Behavior in Continuous Casting of Steel" by Hyunjin Yang, Surya P. Vanka, and Brian G. Thomas.

- "Numerical Simulation on Oxidation of Lanthanum During the Electroslag Remelting Process" by Tianjie Wen, Haijie Zhang, Xiujie Li, Le $\mathrm{Yu}$, Ying Ren, Hong Liu, and Lifeng Zhang. 